\title{
Autologous Anti-CD19/CD22 CAR T-cells AUTO3
}

National Cancer Institute

\section{Source}

National Cancer Institute. Autologous Anti-CD19/CD22 CAR T-cells AUTO3. NCI

Thesaurus. Code C162620.

A preparation of autologous T-lymphocytes that have been transduced with a bicistronic retroviral vector encoding both an anti-CD19 chimeric antigen receptor (CAR) fused to OX40 co-stimulatory domain and an anti-CD22 CAR linked to the intracellular signaling domains of 4-1BB (CD137) and the zeta chain of the TCR/CD3 complex (TCRzeta;

CD247; CD3zeta), optimized with a novel pentameric spacer derived from the collagen oligomeric matrix protein (COMP), with potential antineoplastic activity. Upon administration, the autologous anti-CD19/CD22 CAR T-cells AUT O3 bind to and induce selectivity in tumor cells expressing CD19 and CD22. CD19 and CD22, both transmembrane phosphoglycoproteins expressed on the surface of cells in the B lineage, are often overexpressed on malignant B-cells. By simultaneously targeting two B-cell antigens, this preparation may minimize relapse due to single antigen loss in patients with B-cell malignancies. 